\title{
Comparação da potência anaeróbia entre as posições táticas em jogadores de futebol: estudo retrospectivo
}

\section{Comparison of anaerobic power between tactical positions in soccer players: a retrospective study}

\author{
Tiago Cetolin ${ }^{1}$ \\ Valdeci Foza \\ Juliano Fernandes da Silva \\ Luiz Guilherme Antonacci Guglielmo ${ }^{1}$ \\ Osvaldo Donizete Siqueira ${ }^{2}$ \\ Marcelo Francisco da Silva Cardoso ${ }^{3}$ \\ Luiz Antonio Barcellos Crescente ${ }^{2}$
}

Resumo - O objetivo do estudo foi comparar a Potência anaeróbia máxima (Pmáx); Potência Anaeróbia Média (Pmed) e índice de fadiga (IF) entre atletas de diferentes posições táticas no futebol. Para isso, 248 atletas profissionais de futebol de campo $(25,8 \pm 1,72$ anos, $77,52 \pm 4,90 \mathrm{Kg}$ e $178,48 \pm 5,63 \mathrm{~cm}$ ), pertencentes a $1^{\text {a }}$ e $2^{\text {a }}$ divisão do estado do Rio Grande do Sul, avaliados durante o período de 1999-2010, divididos em cinco posições táticas: goleiros (GO) $(n=28)$, laterais (LA) $(n=35)$, zagueiros (ZA) $(n=54)$, meio-campistas (MC) $(\mathrm{n}=81)$ e atacantes $(\mathrm{AT})(\mathrm{n}=50)$. Os atletas executaram o RAST test para determinar a Pmáx, Pmed e IF. Foi utilizada a ANOVA one way e post hoc de Tukey adotando-se o valor de significância de $\mathrm{p}<0,05$. Diferenças significativas para a Pmáx $(\mathrm{p}<0,05)$ dos LA comparadas aos GO, ZA e MC; da Pmed dos LA para os GO, ZA e MC e dos AT e MC em relação aos $\mathrm{GO}(\mathrm{p}<0,05)$. Na potência relativa de cada sprint, houve manutenção durante o $1^{\circ}$ e $2^{\circ}$ sprint entre as posições, exceto os $\mathrm{MC}$ e durante o $5^{\circ}$ e $6^{\circ}$ sprint, independente da posição, não houve decréscimo do desempenho de potência. Visto que foram encontradas diferenças significativas de Pmáx e da Pmed, dos LA em relação aos GO, ZA e $\mathrm{MC}$, concluímos que esta evidência confirma que atletas de diferentes posições táticas apresentam variação quanto às características de suas ações.

Palavras-chave: Desempenho atlético; Futebol; Testes de aptidão.

Abstract - This study aimed to compare the maximal anaerobic power (Pmáx), mean anaerobic power (Pm) and fatigue index (FI) among soccer players of different tactical positions. For this purpose, 248 professional soccer players $(25.8 \pm 1.72$ years old, $77.52 \pm 4.90$ $\mathrm{kg}$ and $178.48 \pm 5.63 \mathrm{~cm}$ ) from the $1^{\text {st }}$ and $2^{\text {nd }}$ division of the Rio Grande do Sul State, Brazil, participated in this study. They were evaluated between 1999 and 2010 and divided into five positions: goalkeepers $(G K)(n=28)$, external defenders $(E D)(n=35)$, central defenders $(C D)$ $(n=54)$, midfielders $(M F)(n=81)$, and forwards $(F)(n=50)$. The RAST test was performed to evaluate their Pmáx, Pmed, and FI. ANOVA one-way and Tukey's post hoc were used to evaluate the relationship between the variables; significant level was $p<0.05$. Significant differences $(p<0.05)$ were found for Pmáx of ED compared to GK, CD and MF. Similarly, $P m$ of ED also presented significant difference when compared to GK, CD and $M F$, as well as of $F$ and $M F$ compared to $G K(p<0.05)$. In the relative power of each sprint, the athletes maintained the velocity during the $1^{\text {st }}$ and $2^{\text {nd }}$ sprint regardless of their positions, except for $M F$, and during the $5^{\text {th }}$ and $6^{\text {th }}$ sprint. There was no decrease in power performance for all positions. Since significant differences were found in Pmáx and Pm of ED compared to GK, $C D$ and $M F$, we conclude that athletes of different tactical positions presented variation regarding the characteristics of their actions.

1 Universidade Federal de Santa Catarina. Laboratório de Esforço Físico, Programa de Pós-Graduação em Educação Física. Florianópolis, SC. Brasil.

2 Universidade Luterana do Brasil, Laboratório de Pesquisas em Desempenho Humano. Canoas, RS. Brasil.

3 Universidade Federal do Rio Grande do Sul. Escola Superior de Educação Física. Porto Alegre, RS. Brasil.

Recebido em 27/07/12 Revisado em 06/09/12 Aprovado em 11/02/13

Key words:Aptitude tests; Athletic performance; Soccer. 


\section{INTRODUÇÃO}

O futebol é um esporte caracterizado por exercícios intermitentes aleatórios, sendo a capacidade de sprints repetidos (CSR) essencial para o desempenho durante os jogos ${ }^{1}$. Tem sido demonstrado que mesmo representando menos que $10 \%$ da distância percorrida total durante o jogo de futebol, a CSR é considerada um importante componente do desempenho em esportes de equipe.

Recentemente, foi observado que atletas de futebol realizam durante $o$ jogo de futebol de campo em média 17,3 \pm 7,7 sprints $^{2,3}$, distâncias de 19,3 $\pm 3,2$ metros e com velocidades acima de $23 \mathrm{~km} . \mathrm{h}^{-1}$, estando estes fatores correlacionados com aspectos decisivos da partida e ao sucesso da equipe durante a temporada ${ }^{2}$. Além disso, as diferentes posições táticas apresentam variação quanto à distância média dos sprints realizados, no número de solicitações e nas pausas entre os mesmos ${ }^{3,4}$. Os laterais, quando comparados a outras posições táticas, apresentam maior frequência de sprints acima de 20 metros durante o jogo ${ }^{2}$. Também tem sido observado que há uma diferença quanto aos valores de velocidade (sprints) nas diferentes posições táticas. Os atacantes são os que apresentam maiores velocidades nas distâncias de 5, 10 e 20 metros, enquanto os laterais apresentam maiores velocidades em distâncias de 30 e 40 metros. Tais evidências sugerem que cada posição tática possui um nível de solicitação metabólica conforme suas ações, o que gera diferentes adaptações nos processos de produção de energia ${ }^{3,7}$.

Zacharogiannis et al., ${ }^{8}$ propuseram um teste de CSR, denominado Running Anaerobic Sprint Test (RAST), que tem sido frequentemente utilizado para controle por treinadores e preparadores físicos no futebol' ${ }^{9}$. Zagatto et al. ${ }^{10}$ mostraram que o RAST é um procedimento reprodutível e válido para avaliar a potência anaeróbia. Além disso, é reconhecido como um bom indicador do desempenho de corrida ( 35 a 400 metros) e que podem ser facilmente adicionado nas rotinas de treinamento de atletas de vários esportes, como o futebol. Além de fornecer valores da potência anaeróbia, estudos prévios também têm investigado sua relação com a potência muscular ${ }^{9}$, com a aptidão aeróbia ${ }^{11}$ e diferenças entre as categorias de formação no futebol ${ }^{12}$, entretanto, ainda não foi levantado o questionamento do desempenho no RAST test de atletas de posições táticas diferentes, tendo em vista que há a necessidade vigente que treinadores e preparadores físicos possam individualizar as rotinas e cargas de treinamento, utilizando o CSR, conforme a posição tática exercida.

Desta forma, escassos são os estudos na literatura apontando valores de referência com grande número de atletas profissionais de futebol para este protocolo de avaliação, bem como os caracterizando conforme sua posição tática. Assim, o objetivo do estudo foi comparar a potência e a capacidade anaeróbia entre atletas profissionais que exercem diferentes posições táticas no futebol. 


\section{PROCEDIMENTOS METODOLÓGICOS}

\section{Sujeitos}

A seleção dos voluntários do estudo foi de forma intencional, não probabilística, composta por 248 atletas profissionais de futebol de campo com idade, massa corporal total e estatura, respectivamente de 25,8土1,72 anos, 77,52 $\pm 4,90 \mathrm{Kg}$ e $178,48 \pm 5,63 \mathrm{~cm}$ pertencentes a $1^{\text {a }}$ e $2^{\text {a }}$ divisão do estado do Rio Grande do Sul, avaliados durante o período de 1999-2010. Todas as avaliações foram realizadas durante o período preparatório básico e período preparatório específico, em um terreno de grama natural, onde eram realizados treinamentos das equipes e com calçado próprio do esporte (chuteira). Todas as avaliações foram realizadas no período da manhã ( 8-12 horas).

Foram utilizados os seguintes critérios de exclusão:

- Atletas que apresentaram qualquer lesão no mês anterior à avaliação;

- Atletas retornando do departamento médico às rotinas de treinamento;

- Atletas que apresentavam alguma forma de limitação antes das avaliações.

- Foram utilizados os seguintes critérios de inclusão:

- Atletas que não apresentavam nenhuma espécie de limitação para exercícios vigorosos;

- Atletas que realizaram treinamentos de baixa intensidade 24 horas antes da realização das avaliações.

Em relação às posições táticas, os atletas foram divididos em: goleiros (GO), laterais (LA), zagueiros (ZA), Meio-campistas (MC) e atacantes (AT).

\section{Instrumentos e procedimentos}

Antes de iniciarem os procedimentos para a coleta de dados, os atletas que participaram do estudo foram esclarecidos sobre os objetivos e os métodos da pesquisa, para então assinarem o Termo de Consentimento Livre e Esclarecido. O presente estudo foi aprovado pelo Comitê de Ética em Pesquisas com Seres Humanos da Universidade Luterana do Brasil sob o número (105H-2009). Tendo em vista que as coletas de dados iniciaram no ano de 1999, o estudo foi caracterizado como retrospectivo.

Inicialmente, foi aferida a massa corporal dos atletas, utilizando-se uma balança digital (Tanita UM080, Brasil). Após este procedimento e antecedendo as coletas de dados, os jogadores realizaram aquecimento prévio, que foi constituído de quinze minutos de aquecimento e alongamento, seguido por cinco minutos de repouso.

\section{Avaliação da capacidade de sprints repetidos (CSR)}

Para avaliar a CSR, foi utilizado o RAST test (Running-based Anaerobic Sprint Test) proposto por Zacharogiannis et al. ${ }^{8}$, sendo, recentemente, apresentado sua validade ${ }^{10}$. O RAST consiste em realizar 6 sprints má- 
ximos de $35 \mathrm{~m}$, em um campo de futebol, com intervalo de 10 segundos de pausa passiva entre os sprints. Na realização do teste, foram utilizadas duas fotocélulas eletrônicas (CEFISE, Brasil), dispostas a $35 \mathrm{~m}$ de distância, para capturar os tempos dos sprints. Para fins de análise, utilizaram-se os tempos adquiridos em cada sprint (T1, T2, T3, T4, T5 e T6). Assim, com os tempos adquiridos, foi calculada a potência de cada sprint.

A potência (W) para cada esforço foi obtida por meio do produto entre a massa corporal total do atleta $(\mathrm{kg})$ e a distância de cada esforço elevada ao quadrado $(35 \mathrm{~m})^{2}$. O resultado foi divido pelo tempo de cada esforço elevado ao cubo $\left(\mathrm{T}^{3}\right)^{11}$ demonstrado na equação 1 .

\section{Equação 1:}

$$
\text { Potência }(\mathrm{W})=\left(\left(\mathrm{MC} \times 35^{2}\right) / \mathrm{T}^{3}\right)
$$

Foram registrados como parâmetros anaeróbios do RAST a potência máxima (Pmáx, maior potência entre os 6 esforços), potência média (Pmed, média entre as potências dos 6 esforços), índice de fadiga (equação 2):

\section{Equação 2:}

Índice de Fadiga (IF) $(\%)=\left[\left(\sum \text { tempos } / 6^{\star} \text { Melhor tempo }\right)-1\right]^{\star} 100$

As equações foram utilizadas conforme trabalhos de Dal Pupo et al. ${ }^{9} \mathrm{e}$ Barbero et al. ${ }^{13}$ Depois de encontrados os valores de potência (W) em cada esforço, estes foram divididos pela massa corporal total (MC) do atleta para que fossem encontrados os valores de potência relativos à massa corporal total (W/Kg. $\left.{ }^{-1}\right)$ ao qual foi utilizado como parâmetros anaeróbios do RAST.

\section{Análise estatística}

Utilizou-se a estatística descritiva para análise e tratamento dos dados, apresentados em forma de média e desvio padrão. Para homogeneidade das variâncias, foi utilizado o teste de Levene no intuito de verificar se a homogeneidade das variâncias era atendida.

Para comparar os resultados obtidos em relação à Pmáx, Pmed e IF entre as posições, a potência relativa em cada posição tática e a potência relativa entre todas as posições, foi utilizado o teste de análise de variância One-way, complementado pelo teste de Tukey. Para verificar a normalidade do resíduo padronizado, foi utilizado o teste de normalidade de Kolmogov Smirnov. Foi adotado um nível de significância de 5\%. Para tais mensurações, foi utilizado o pacote estatístico SPSS ${ }^{\circledR} 15.0$ for Windows.

\section{RESULTADOS}

Na tabela 1, apresentam-se valores médios da massa corporal total, da Pmáx e Pmed em (WK.g ${ }^{-1}$ ) e o IF (\%) entre as diferentes posições durante o RAST test. 
Tabela 1. Média e desvio-padrão da massa corporal total (kg) e das variáveis Pmáx, Pmed e IF encontradas no RAST test.

\begin{tabular}{lccccc}
\hline $\begin{array}{l}\text { Posições } \\
(\mathrm{n}=248)\end{array}$ & $\begin{array}{c}\text { Massa Corpo- } \\
\text { ral Total }(\mathrm{kg})\end{array}$ & $\begin{array}{c}\text { Estatura } \\
(\mathrm{cm})\end{array}$ & $\begin{array}{c}\text { Pmáx } \\
(\text { WK.g }\end{array}$ & $\begin{array}{c}\text { Pmed } \\
\left(\text { WK. } \mathrm{g}^{-1}\right)\end{array}$ & $\begin{array}{c}\text { IF } \\
(\%)\end{array}$ \\
\hline GO $(\mathrm{n}=28)$ & $85,65 \pm 6,5$ & $187,60 \pm 1,6$ & $9,90 \pm 1,8$ & $7,62 \pm 1,1^{\#+}$ & $9,86 \pm 3,3^{\#+}$ \\
LA $(\mathrm{n}=35)$ & $70,73 \pm 4,9$ & $175,80 \pm 4,7$ & $11,76 \pm 1,8^{*+\#}$ & $9,32 \pm 1,3^{*+\#}$ & $8,72 \pm 2,2$ \\
ZA $(\mathrm{n}=54)$ & $78,67 \pm 6,5$ & $185,27 \pm 2,7$ & $10,22 \pm 1,6$ & $8,22 \pm 1,2$ & $8,15 \pm 2,8$ \\
MC $(\mathrm{n}=81)$ & $74,90 \pm 7,5$ & $176,14 \pm 4,5$ & $10,63 \pm 1,5$ & $8,57 \pm 1,2$ & $7,94 \pm 2,8$ \\
AT $(\mathrm{n}=50)$ & $77,69 \pm 6,9$ & $179,36 \pm 6,2$ & $10,82 \pm 2,0$ & $8,66 \pm 1,5$ & $8,33 \pm 3,0$ \\
\hline
\end{tabular}

${ }^{*} p<0,05$ em relação a as $\mathrm{G0}$; $\uparrow p<0,05$ em relação aos $Z A ; \# p<0,05$ em relação aos $M C ;+p<0,05$ em relação $\operatorname{aos} \mathrm{AT}$

Tabela 2. Valores médios da potência anaeróbia máxima relativa (WK.g-1) durante os sprints realizados no RAST test.

\begin{tabular}{|c|c|c|c|c|c|}
\hline $\begin{array}{l}\text { Variáveis } \\
(n=248)\end{array}$ & $\begin{array}{c}\mathrm{GO} \\
(n=28)\end{array}$ & $\begin{array}{c}\mathrm{LA} \\
(\mathrm{n}=35)\end{array}$ & $\begin{array}{c}\text { ZA } \\
(n=54)\end{array}$ & $\begin{array}{c}M C \\
(n=81)\end{array}$ & $\begin{array}{c}\text { AT } \\
(n=50)\end{array}$ \\
\hline P1 $\left(\right.$ WK.g $\left.{ }^{-1}\right)$ & $9,76 \pm 1,82^{\#+\& \S}$ & $11,59 \pm 1,8^{\#+\& 5}$ & $10,09 \pm 1,7^{\#+\& \S}$ & $10,52 \pm 1,6^{t \#+\& \S}$ & $10,62 \pm 1,9^{\#+8.5}$ \\
\hline P2 (WK.g $\left.{ }^{-1}\right)$ & $8,81 \pm 1,78^{\#+\& \S}$ & $10,95 \pm 1,8^{\#+\& \S}$ & $9,39 \pm 1,6^{\#+\& \S}$ & $9,59 \pm 1,8^{\#+\& \S}$ & $9,95 \pm 1,9^{\#+\& \S}$ \\
\hline P3 (WK.g $\left.{ }^{-1}\right)$ & $7,66 \pm 1,4^{\& \S}$ & $9,69 \pm 1,6^{+\& 5}$ & $8,42 \pm 1,5^{\& \S}$ & $8,71 \pm 1,4$ & $8,88 \pm 1,7$ \\
\hline P4 (WK.g $\left.g^{-1}\right)$ & $7,07 \pm 1,1$ & $8,83 \pm 1,3^{\& \S}$ & $7,69 \pm 1,2^{\& \S}$ & $7,96 \pm 1,2^{\S}$ & $7,33 \pm 1,6^{5}$ \\
\hline P5 (WK.g $\left.{ }^{-1}\right)$ & $6,17 \pm 0,8$ & $7,69 \pm 1,2$ & $7,05 \pm 1,1^{\S}$ & $7,43 \pm 1,3$ & $7,11 \pm 1,3$ \\
\hline P6 (WK.g $\left.{ }^{-1}\right)$ & $6,23 \pm 1,0$ & $7,45 \pm 1,2$ & $6,66 \pm 1,1$ & $7,19 \pm 1,2$ & $8,66 \pm 2,1$ \\
\hline
\end{tabular}

tp $<0,05$ em relação a $P 2 ; \# p<0,05$ em relação a $P 3 ;+p<0,05$ em relação a $P 4 ; \& p<0,05$ em relação a $P 5 ; ~ § p$ $<0,05$ em relação a P6

Na tabela 2, estão expostos os valores médios da potência relativa à massa corporal (WK.g-1) das seis repetições do RAST test entre as posições.

Quando comparadas as potências relativas de cada sprint (WK.g $\left.{ }^{-1}\right)$ dos 6 sprints obtidos no RAST test, verificou-se diferença significativa $(\mathrm{p}<0,01)$ entre os mesmos $\left(\mathrm{P} 1=10,51 \pm 1,84\left(\mathrm{WK} . \mathrm{g}^{-1}\right), \mathrm{P} 2=9,76 \pm 1,77\right.$ (WK.g$\left.{ }^{1}\right), P 3=8,70 \pm 1,63\left(W_{K} \cdot g^{-1}\right), P 4=7,95 \pm 1,37\left(W K . g^{-1}\right), P 5=7,22 \pm 1,35\left(W_{K} \cdot g^{-1}\right)$ e P6=6,98 $\pm 1,25$ (WK.g $\left.{ }^{-1}\right)$, com exceção entre P5 e P6 (p = 0,543), conforme apresentado na figura 1 .

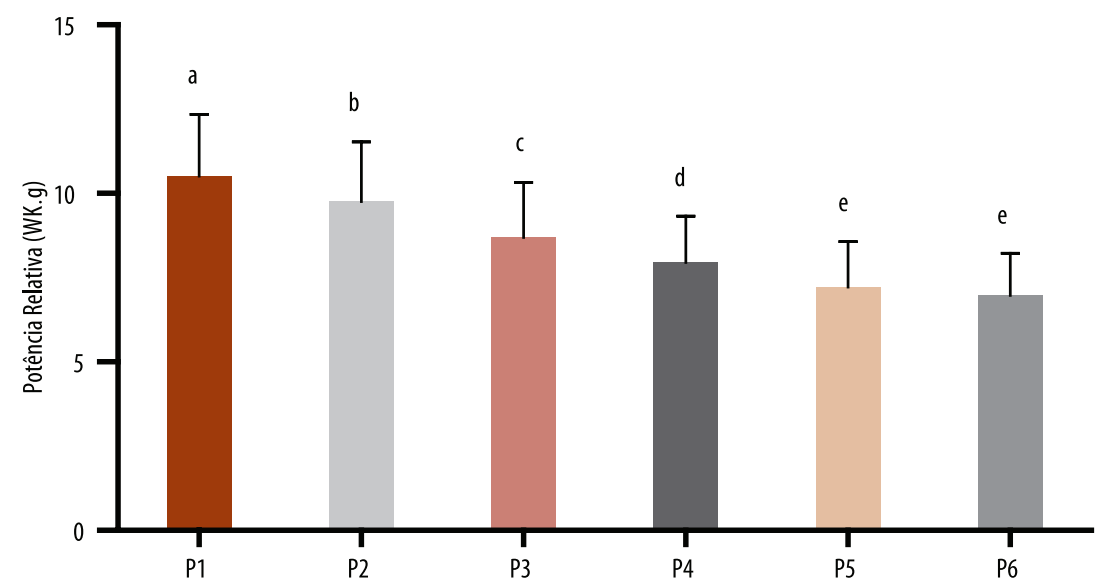

Figura 1 - Comparação entre o tempo obtido nos seis sprints (P1, P2, P3, P4, P5 e P6) de todas as posições. (Nota: letras diferentes mostram diferença significativa, $p=0,00$ ) 


\section{DISCUSSÃO}

O principal achado do presente estudo foram as diferenças significativas encontradas de Pmáx dos LA, em relação aos GO ( $\mathrm{p}=0,00), \mathrm{ZA}(\mathrm{p}=0,00)$ e $\mathrm{MC}(\mathrm{p}=0,01)$ e da Pmed, dos LA comparado aos GO $(\mathrm{p}=0,00), \mathrm{ZA}(\mathrm{p}=0,00)$ e $\mathrm{MC}(\mathrm{p}=0,03)$. Esta evidência confirma que atletas de diferentes posições táticas apresentam variação quanto às características de suas ações ${ }^{3,4}$. As diferentes solicitações inerentes aos treinamentos e jogos tendem a contribuir para estes resultados, visto que as adaptações metabólicas ao treinamento de CSR são dependentes da duração de cada sprint, recuperação entre repetições, volume e frequência de séries de treinamento ${ }^{14}$.

Os maiores valores de Pmáx nos LA podem ser explicados em função do elevado número de sprints que estes realizam durante o jogo, em relação às outras posições ${ }^{4}$, bem como um número superior de sprints acima de 20 metros $^{2}$, assemelhando-se, assim, ao estímulo utilizado no protocolo do presente estudo (35 metros).

Pasquareli et al. ${ }^{6}$ observaram que os LA apresentaram maiores velocidades quando comparados com outras posições em corridas com distância (40 metros) similar ao protocolo de avaliação do RAST test. No presente estudo, os LA obtiveram os melhores resultados na Pmáx o que demonstra uma resposta específica nesta variável conforme a posição tática exercida. Isso sugere que a CSR pode ser desenvolvida tanto por meio de rotinas específicas de treinamento, como por treinamentos que utilizem prioritariamente as situações ocorridas no jogo de futebol ${ }^{1}$.

A Pmed dos LA obtidas durante os sprints repetidos pode ser explicada por uma adaptação específica às ações desta posição tática, pois em virtude de atuarem tanto defensivamente como ofensivamente ${ }^{4,15,16}$ necessitam realizar um maior volume de exercício em alta intensidade e percorrerem maiores distâncias em sprint durante treinamentos e competições ${ }^{17}$. Em recente estudo com análise de jogos do campeonato europeu de futebol de campo $^{16}$, foi demonstrado que os AT e LA são as posições táticas que percorrem a maior distância total em sprint e realizam o maior número de sprints acima de 10 metros $^{16}$, o que justifica não termos encontrado, no presente estudo, diferenças significativas entre os LA e AT na Pmed.

Sendo assim, a incapacidade de manter o desempenho em sprints repetidos tem sido atribuída, principalmente, ao acúmulo de $\mathrm{H}^{+18}$, depleção de fosfocreatina muscular ${ }^{19} \mathrm{e}$ fatores como mudanças na coordenação neuromuscular da contração muscular ${ }^{20}$. Foram observados, em vários estudos, valores médios de concentrações de lactato [la] encontrados após a realização do RAST test em torno de $\left(9,81 \text { mmol.L } \mathrm{L}^{-1}\right)^{11}$ de $(10,12 \pm 1,48$ mmol. $\left.\mathrm{L}^{-1}\right)^{9}$ e de $\left(14,23 \pm 2,68 \mathrm{mmol} . \mathrm{L}^{-1}\right)^{10}$, chegando a valores máximos de até $\left(19 \mathrm{mmol} . \mathrm{L}^{-1}\right)^{10}$. Além destas [la] não refletirem os achados encontrados em jogos de futebol de campo ${ }^{17}$, nem sempre as mensurações das [la] são realizadas após períodos intensos do jogo o que dificulta a suposição dos valores reais de [la] encontrados durantes os jogos e sua comparação com os valores encontrados após um teste de CSR. Em adição, estes altos 
valores encontrados (19 mmol. $\left.\mathrm{L}^{-1}\right)$ dos estudos supracitados não tiveram como sujeitos atletas de futebol de campo o que pode explicar estes altos valores de [la].

Assim, durante estas ações consideradas láticas, está relatado que há elevada formação de íons $\mathrm{H}^{+}$, ocorrendo redução no $\mathrm{pH}$ intramuscular, o que irá limitar a atividade glicolítica pela inibição da fosfofrutoquinase, que é a enzima-chave regulatória das enzima-chave regulatória da glicólise ${ }^{18}$, embora tenha sido relatado que os aumentos da concentração de lactato contribuem para o tamponamento dos íons $\mathrm{H}^{+21}$.

Outra hipótese é que os laterais estão entre os atletas que apresentam o condicionamento aeróbio mais desenvolvido entre as posições táticas do futebol ${ }^{22,23} \mathrm{o}$ que pode trazer benefícios a CSR, já que em recente estudo foi evidenciada a importância da aptidão aeróbia na CSR ${ }^{24}$. Essa hipótese é corroborada pelo aumento da participação aeróbia com o aumento da distância do sprint ${ }^{25}$.

As diferenças significativas encontradas entre os GO com os AT $(\mathrm{p}=0,00)$ e $\mathrm{MC}(\mathrm{p}=0,01)$ podem ser explicadas pela característica das ações dos GO durante o jogo de futebol, que apresenta poucas situações que envolvam sprints repetidos ${ }^{1}$. Esta evidência também pode ser observada na avaliação da variável IF que apresentou diferenças significativas $(\mathrm{p}=0,02)$ entre os GO e MC.

Os resultados do IF estão elevados quando comparados aos de um recente estudo com jogadores que obtiveram média de $6,79 \pm 1,41 \%{ }^{9}$. Sendo assim, valores de IF acima de $10 \%$ apontam que o atleta precisa aprimorar sua tolerância aos esforços anaeróbios intermitentes e que estaria sob os efeitos deletérios da fadiga ${ }^{8}$. Além disso, foram verificadas em cada posição tática as diferenças de potência relativa (WK.g ${ }^{-1}$ ) durante os seis sprints do RAST test. Verificou-se um decréscimo no desempenho dos atletas no decorrer dos 6 sprints. Tal fenômeno parece acontecer em séries repetidas de exercício intenso, já que, à medida que aumenta o tempo de exercício há uma diminuição na contribuição do metabolismo glicolítico para a ressíntese do ATP e aumento da participação do metabolismo aeróbio ${ }^{13}$ Apesar da predominância anaeróbia na CSR, o esforço final pode ser mantido em função do aumento da participação do sistema aeróbio, que parece responder rapidamente à demanda energética ao final de exercícios intensos.

Gaitanos et al. ${ }^{19}$, observaram que após a realização de 10 sprints de 6 segundos, em um cicloergômetro, com 30 segundos de intervalo de recuperação, foram observadas significativas reduções da contribuição glicolítica no fornecimento energético no $10^{\circ}$ sprint em relação ao primeiro. Desta forma, os autores postulam que, durante os últimos sprints, $a$ contribuição da glicólise é diminuída, sendo o ATP regenerado pelas fontes de PCr, restauradas, parcialmente, no intervalo, e pelo aumento da participação do metabolismo aeróbio.

Verificou-se, ainda, uma capacidade de manutenção da potência relativa (WK.g ${ }^{-1}$ ) do P1 para o P2, exceto os MC (tabela 2), o que se presume que durante estes dois sprints foi possível utilizar energia fornecida pelos 
estoques de PCr e glicogênio muscular, tendo em vista que, durante uma única repetição de corrida de curta duração, o ATP é ressintetizado, principalmente, por uma combinação de $\mathrm{PCr}$ e degradação do glicogênio muscular, com uma contribuição mínima do metabolismo aeróbio ${ }^{19}$.

Em relação do P2 para o P3 (tabela 2), exceto os GO, foram encontradas diferenças significativas em todas as posições $(\mathrm{p}=0,00)$. Tal evidência sugere que independente da posição há uma redução considerável na potência a partir do P3, o que pode ser planificada por treinadores e preparadores físicos durante a montagem e a utilização de rotinas de treinamento a partir dos parâmetros utilizados no RAST test. A posição tática que necessita percorrer sprints em uma maior distância durante o jogo e que realizam com mais frequência estas ações durante o jogo de futebol, como os LA, podem ser treinadas utilizando o modelo do RAST test. Encontramos diferenças entre a potência relativa nos seis sprints máximos, desta forma, nossos resultados apontam que atletas de todas as posições, exceto os MC, são capazes de manter dois sprints repetidos na mesma capacidade durante o teste.

A duração da recuperação tem uma contribuição importante sob esta redução de desempenho ${ }^{26}$. Fernandes da Silva et al., ${ }^{24}$ utilizando um protocolo com ( 7 x 34,2 metros) ${ }^{27}$, observaram diminuição significativa no desempenho a partir do quarto sprint, tendo em vista que a recuperação entre os sprints deste protocolo é de 25 segundos, diferentemente do modelo do RAST test (10 segundos) utilizado no presente estudo, que obtiveram queda de desempenho a partir do terceiro sprint. Assim, o curto período de recuperação do modelo do RAST test colabora para queda considerável dos fosfagênios e um aumento da contribuição da glicólise anaeróbia e do sistema aeróbio de fornecimento de energia ${ }^{26}$. Isto pode ter sido mais evidente em atletas que apresentaram maiores valores de Pmáx como os LA. Hirvonen et al., ${ }^{28}$ observaram em grupo de velocistas de elite, que os atletas com maiores velocidades depletavam seus estoques de PCr em uma maior taxa que os corredores com menores velocidade do mesmo grupo, o que sugere que além da maior participação de PCr há uma menor taxa de ressíntese nos sprints subsequentes.

No P5 e P6 (tabela 2), verificou-se uma capacidade de manutenção da

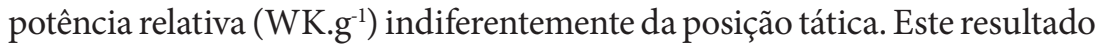
é semelhante a um estudo anterior, utilizando o RAST test ${ }^{9}$, o qual apontou que o esforço final pode ser mantido graças ao aumento da participação do sistema aeróbio, que parece responder rapidamente a demanda energética ao final de exercícios intensos ${ }^{29}$. Em adição, quando os sprints são repetidos, há a inibição progressiva da glicólise, cujos efeitos são parcialmente compensadas por um aumento gradual na ressíntese aeróbia de ATP ao longo dos sucessivos sprints ${ }^{18}$.

Por fim, como limitação do estudo não foram apresentados os valores de percentual de gordura dos atletas avaliados os quais poderiam influenciar nos resultados encontrados. 


\section{CONCLUSÃO}

Desta forma, conclui-se que os LA apresentam os maiores valores de Pmáx e de Pmed, em relação a outras posições, devido a adaptação específica das ações desta posição tática.

Por fim, em todas as posições táticas há uma redução considerável na potência a partir do terceiro sprint, o que pode ser planificada por treinadores e preparadores físicos durante a montagem e a utilização de rotinas de treinamento a partir dos parâmetros utilizados no RAST test.

\section{REFERENCIAS BIBLIOGRÁFICAS4}

1. Kaplan, T. Examination of repeated sprinting ability and fatigue index of soccer players according to their positions. J Strength Cond Res 2010;24:1495-501.

2. Di Salvo V, Gregson W, Atkinson G, Tordoff P, Drust B. Analysis of High Intensity Activity in Premier League Soccer. Int J Sports Med 2009;30:205-12.

3. Di Salvo V, Baron R, Tschan H, Calderon Montero FJ, Bachl N, Pigozzi F. Performance characteristics according to playing position in elite soccer. Int J Sports Med 2007;28(3): 222-7.

4. Di Salvo, V, Baron R, Gonzalez-Haro C, Gormasz C, Pigozzi F, Bachl N. Sprinting analysis of elite soccer players during European Champions League and UEFA Cup matches. J Sports Sci 2010;3:1-6.

5. Sporis G, Jukic I. Fitness profiling in soccer: physical and physiologic characteristics of elite players. J Strength Cond Res 2009;23(7):1947-53.

6. Pasquarelli BN, Stanganelli LCR, Dourado AC, Loch MR, Andrade CA. Análise da velocidade linear em jogadores de futebol a partir de dois métodos de avaliação. Rev Bras Cineantropom Desempenho Hum 2009;11(4):408-14.

7. Reilly T. Energetics of high-intensity exercise (soccer) with particular reference to fatigue. J Sport Sci 1997;15(3):257-63.

8. Zacharogiannis E, Paradisis G, Tziortzis S. An evaluation of tests of anaerobic power and capacity. Med Sci Sports Exerc 2004; Supp 36(5):116.

9. Dal Pupo J, Almeida CMP, Detanico D, Da Silva JF, Guglielmo LG, Santos SG. Potência muscular e capacidade de sprints repetidos em jogadores de futebol. Rev Bras Cineantropom Desempenho Hum 2010;12(4): 255-61.

10. Zagatto AM, Beck WR, Gobatto CA. Validity of the running anaerobic sprint test for assessing anaerobic power and predicting short-distance performances. J Strength Cond Res 2009;23(6):1820-7.

11. Roseguini AZ, Silva, ASR, Gobatto, CA. Determinações e relações dos parâmetros anaeróbios do RAST, do limiar anaeróbio e da resposta lactacidemica obtida no início, no intervalo e ao final de uma partida oficial de handebol. Rev Bras Med Esporte 2008;14: 46-50.

12. Spigolon LM, Borin JP, Leite GS, Padovani CRP. Potência anaeróbia em atletas de futebol de campo: diferenças entre categorias. Pesq Educ Fís 2007;(6):421-8.

13. Barbero JC, Méndez A, Bishop D. La capacidad para repetir esfuerzos máximos intermitentes: aspectos fisiológicos. Arch Med Deporte 2006; 23(115):379-89.

14. Ross A, Leveritt M. Long-term metabolic and skeletal muscle adaptations to short-sprint training: implications for sprint training and tapering. Sports Med 2001;31:1063-82.

15. Abbasian S, Golzar S, Onvani V, Sargazi L. The predict of RAST Test from WANT test in Elite Athletes. Res J Recent Sci 2012;1(3):72-5.

16. Goulart LF, Dias RMR, Altimari LR. Força Isocinética de Jogadores de futebol categoria sub-20: compração entre diferentes posições de jogo. Rev Bras Cineantropom Desempenho Hum 2007;9(2):165-9. 
17. Morh M, Krustrup P, Bangsboo J. Match performance of high-standard soccer players with special reference to development of fatigue. J Sports Sci 2003;21:519-28.

18. Bortolotti H, Pasquarelli BN, Caldeira LFS, Altimari LR, Nakamura FY. Avaliação da capacidade de realizar sprints repetidos no futebol. Motriz 2010;16(4):1006-12.

19. Gaitanos GC, Williams C, Boobis LH, Brooks S. Human muscle metabolism during intermittent maximal exercise. J Appl Physiol 1993;75(2):712-9

20. Mendez-Villanueva A, Hamer P, Bishop D. Fatigue Responses during repeated sprints matched for initial mechanical output. Med Sci Sport Exerc 2007;39:2219-25.

21. Bertuzzi RCM, Lima Silva AE, Abad C, Pires FO. Metabolismo do lactato: uma revisão sobre a bioenergética e a fadiga muscular Rev Bras Cineantropom Desempenho Hum 2009;11(2):226-34.

22. Santos JAR. Estudo comparativo, fisiológico, antropométrico e motor entre futebolistas de diferente nível competitivo. Rev Paul de Educ Fís 1999;13(2):146-59.

23. Balikian P, Lourenção A, Ribeiro LFP, Festuccia WTL, Neiva CM. Consumo máximo de oxigênio e limiar anaeróbio de jogadores de futebol: comparação entre as diferentes posições. Rev Bras Med Esporte 2002;8(2):32-6.

24. Fernandes da Silva J, Guglielmo LGA, Bishop D. Relationship between different measures of aerobic fitness and repeated-sprint ability in elite soccer players. J Strength Cond Res 2010;(24):2115-21.

25. Balsom P, Seger J, Sjodin B, et al. Maximal-intensity intermittent exercise: effect of recovery duration. Int J Sports Med 1992;(13):528-33.

26. Little T, Williams AG. Effects of sprint duration and exercise: Rest ratio on repeated sprint performance and physiological responses in professional soccer players. J Strength Cond Res 2007;21(2):646-8.

27. Bangsbo J. Fitness Training for Football: A scientific approach. HO+Storm, Bagsvaerd, 1994.

28. Hirvonen J, Rehunen S, Rusko H. Breakdown of high energy phosphate compounds and lactate accumulation during short supramaximal exercise. Eur J Appl Physiol 1987;(56):253-9.

29. Duffield R, Dawson B, Goodman C. Energy system contribution to 400- and 800-metres track running. J Sports Sci 2005;23(3):299-307.

\section{Endereço para correspondência}

Tiago Cetolin

Universidade Federal de Santa

Catarina

Centro de Desportos - Laboratório de Esforço Físico

Campus Universitário da Trindade, $\mathrm{s} / \mathrm{n}$

CEP: 88040-900 Florianópolis, SC.

Brasil

E-mail: tcetolin@terra.com.br 